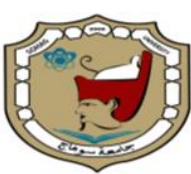

Sohag University

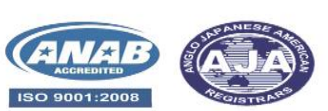

Sohag Medical Journal
Faculty of Medicine

\title{
Superficial cryotherapy, does it work in alopecia areata?
}

\author{
Essam El-Din Abd-El Aziz Nada, Hanan Abdel Rady Assaf, Soha \\ Hishmat Aboeldahab, Zeinab Abu-El Baha Gouda.
}

Department of Dermatology, Venereology, and Andrology, Faculty of Medicine, Sohag University.

\begin{abstract}
:
Alopecia areata is a common cause of nonscarring alopecia, with patchy, confluent, or diffuse patterns, involving mainly the scalp and other hairy areas of the body. It's considered a therapeutic challenge due to prognosis, unpredictable course, and variable efficacy of available therapies. Several studies have evaluated the efficacy of cryotherapy in the treatment of alopecia areata with varying success rates.

Superficial cryotherapy using liquid nitrogen mainly was used in several studies for treating alopecia areata with various subtypes including mild, moderate, and recalcitrant alopecia areata, and alopecia totalis with variable rates of therapeutically accepted terminal hair regrowth. Its main mechanisms of efficacy in alopecia areata are vascular changes and immunomodulation.

Superficial cryotherapy was effective and safe in treating alopecia areata of the eyebrows. It was nearly as effective as topical steroids and topical PUVA therapy and was combined with topical immunotherapy and topical steroid with increased efficacy. It's a well-tolerated، convenient, and simple office-based procedure، especially in children who are susceptible to side effects of other conventional therapeutic options. The reported side effects of superficial cryotherapy included vesiculation, erosion, crust formation, transient pigmentary alteration, and partial leukonychia, however, they were transient.
\end{abstract}

\section{Introduction:}

Alopecia areata (AA) is the most prevalent autoimmune disorder and the second most frequent cause of hair loss after androgenetic alopecia ${ }^{(1)}$. It's a complex inflammatory disease characterized by cellular infiltration of $\mathrm{T}$ lymphocytes targeting hair follicles (HFs), disrupting the anagen phase ${ }^{(2)}$. It affects $1-2 \%$ of the general population, with a lifetime risk of $1.7 \%^{(3)}$. Depression, generalized anxiety, posttraumatic stress disorder, social phobia, and suicidal thoughts are associated with $\mathrm{AA}^{(4)}$. Available treatment options for AA inc-lude topical immunotherapy, topical and intralesional corticosteroids, PUVA therapy, systemic immunosuppressants such as systemic corticosteroids, methotrexate, cyclosporine A and azathioprine (5), biologic agents such as IL-12/IL-23 p40 blockers ${ }^{(6)}$ and Janus kinase (JAK) inhibitors ${ }^{(7)}$. None of the traditional immune-suppressive agents and immunologically targeted newer therapies could establish long-lasting remission ${ }^{(8)}$. No systemic agents are approved by the FDA for the treatment of AA ${ }^{(9)}$. Superficial cryotherapy is considered as one of the emerg-ing unconventional therapies for AA ${ }^{(10)}$. Cryotherapy is a controlled and targeted tissue destruction by applying a cold temperature substance (11). It's performed using a cryogen to cool the targeted tissue to subzero 
temperatures. The cry-ogens used include liquid nitrogen $\left(-196^{\circ} \mathrm{C}\right)$, carbon dioxide snow $\left(-79^{\circ} \mathrm{C}\right)$, nitrous oxide $\left(-70^{\circ} \mathrm{C}\right)$, dimethyl ether and propane $\left(-57^{\circ} \mathrm{C}\right)$, and salt-ice mixture $\left(-20^{\circ} \mathrm{C}\right)$ ${ }^{(12)}$. Liquid nitrogen is the typical cryogen. It's only used under the clinician's supervision to avoid unnecessary skin damage ${ }^{(13)}$. There are prescription-free cryogenic devices, which freeze through evaporative cooling by the sudden expansion of compressed solvents, such as dimethyl ether (DME) and the mixture of $75 \%$ and propane $25 \%$ $(\mathrm{DMEP})^{(14,15)}$. The extent of tissue injury induced by cryotherapy is determined by the rate of freezing, the coldest temperature reached, the freeze tim$\mathrm{e}$, and the rate of thawing (12)

Superficial cryotherapy refers to tissue exposure to hypothermic cryogen for a limited period of few seconds, unlike the conventional standard cryotherapy where the exposure is prolonged till obtaining an ice crystal. which can cause cell disruption and rupture due to direct effect or osmotic effect ${ }^{(16,17)}$, and also causes vascular damage vascular constriction, platelet aggregation، thrombus formation occluding tissue blood flow, ischemia, increased permeability, apoptosis, and immunological effect ${ }^{(16)}$.

With this limited exposure to the cryogen in superficial cryotherapy, neither crystal formation nor blood flow occlusion occurs ${ }^{(18)}$. Superficial cryotherapy induces hair regrowth in the AA through those mechanisms:

After initial vasoconstriction induced by cooling, during the thaw period as the temperature reaches zero-degree Celsius, significant local vasodilatation occurs, with increased blood flow to the tissues, followed by reactive vasodilatation, improving the microcirculation ${ }^{(18)}$. The local edema and inflammation following superficial cryotherapy may play a role in inducing vasodilatation ${ }^{(19)}$.
Superficial cryotherapy is suggested to partially damage or denature keratinocytes, especially the antigenic components of the HF keratin 16 and trichohyalin which are targeted by antibodies, this decreases the perifollicular cellular infiltrates ${ }^{(20)}$. Superficial cryotherapy alters immune-logic processes by decreesing the function of Langerhans cells which are already have increased numbers in progressive AA, and this further decreases the $T$ cell infiltration ${ }^{(20)}$.

Cryotherapy and hypothermia have been associated with reduced in vitro and in vivo T-cell and monocyte activeation response, reduced IL-17 release in $\mathrm{T}$ cells, reduced IL-1 $\beta / \mathrm{IL}-23$ activation of $\mathrm{T}$ cells, and reduced granzyme B production ${ }^{(21)}$. Abnormal melanocytes wit$\mathrm{h}$ large and bizarre melanosomes were observed around the HFs of AA patients. So, superficial cryotherapy may exert its therapeutic effects on AA by blocking a pathology associated with these abnor-mal melanocytes, or it causes melan-ocyte alteration and prevents their role in the initiation of AA. (22). Superficial cryotherapy using liquid nitrogen was introduced by Huang et al (23). and has been used as a considerable minimally invasive therapeutic modality for AA. The therapeutic efficacy of superficial cryotherapy in AA has been studied by various studies where:

Lei et al ${ }^{(24)}$ reported $>60 \%$ new hair regrowth in $70 / 72(97.2 \%)$ of the patients, considering superficial cryotherapy effective, especially for mild AA, with short treatment duration and minimal adverse effects. Kim et al ${ }^{(25)}$ reported an overall clinical response of $66.7 \%$ in 33 patients with AA.

Also, Lee et al ${ }^{(22)}$ reported an accepted clinical response in $17 / 19(89.5 \%)$ of AA patients, Hong et al ${ }^{(26)}$ reported an overall clinical response in $105 / 153$ $(68.6 \%)$ of AA patients, after 12 weeks of using liquid nitrogen for 3-4 cycles, of 2-3 seconds each, and Faghihi and 
Radan ${ }^{(27)}$ reported an overall response of $80 \%$ in 38 patients with AA using liquid nitrogen once a week for 12 weeks.

In the study of Jun et al ${ }^{(28)}, 353$ patients with AA were treated using liquid nitrogen for 3-4 cycles, of 2-3 seconds each, with $215(60.9 \%)$ of the patients being responders. Amira et al (29), reported a response rate of $60-90 \%$ terminal hair regrowth, in 40/120 (33.3\%) of AA patients.

Additionally, Abdel-Majid et al (30) reported an accepted therapeutic response ( $>50 \%$ terminal hair regrowth in $55 \%$ of AA lesions using liquid nitrogen once weekly for 6 weeks, while Sayed et al ${ }^{(31)}$, obtained $>50 \%$ terminal hair regrowth in $62.9 \%$ of 27 patients with AA using liquid nitrogen in 3-4 freeze-thaw cycles, of 2-3 seconds each, every 2 weeks for 6 sessions.

For recalcitrant AA, Zawar and Karad (19) obtained $>50 \%$ terminal hair regrowth in $8 / 10(80 \%)$ of patients with recalcitrant AA. Owing to its nondestructive and minimally painful nature, superficial cryotherapy was therapeutically effective in treating AA in fragile areas other than the scalp like eyebrows ${ }^{(32,33)}$. Superficial cryotherap$y$ was combined with other therapeutic modalities for AA treatment, such as prednicarbate $0.25 \%$ solution, with a mean SALT score (Severity Alopecia Tool, it deter-mines AA severity based on hair loss percentage) decrease percentage of $40.7 \%$ versus $9.6 \%$ with prednicarbate alone, ${ }^{(34)}$ and with topical immunotherapy using squaric acid dibutyl ester (SADBE) with more efficacy than SADBE alone ${ }^{(35)}$. Compared to other therapeutic modalities of AA, superficial cryotherapy resulted in $(>50 \%)$ terminal hair regrowth in $56.5 \%$ compared to $62.5 \%$ with $0.1 \%$ betamethasone lotion, in 40 patients with 120 recalcitrant AA patches (36), and it had a lower therapeutic effect of
$80 \%$ than $91.5 \%$ achieved by topical clobetasol propionate for patchy recalcitrant AA ${ }^{(27)}$. Also, it was inferior to intralesional triamcinolone injection with a regrowth rate $(>50 \%)$ in $23.3 \%$ and $56.7 \%$ of patients respectively ${ }^{(29)}$. Superficial cryotherapy was less effecttive than topical PUVA therapy for mild-to-moderate AA with $>50 \%$ terminal hair regrowth rates of $62.96 \%$ and $80 \%$ respectively ${ }^{(31)}$. Regarding the superficial cryotherapy technique; Lei et al ${ }^{(24)}$ used a cotton swab soaked with liquid nitrogen in two freeze-thaw cycles, of 2-3 seconds free-ze and 2-3 seconds thaw each, once weekly for 4 weeks. Similarly, Gita and Mohammadreza (36), used a short duration of 2-3 seconds of freeze and 3-5 seconds of a thaw; and Jun et al ${ }^{(28)}$, sprayed liquid nitrogen 3-4 times on an ea-ch patch for 2-3 seconds every 2 weeks.

Zawar and Karad ${ }^{(19)}$, delivered liquid nitrogen via a brass spray tip in 2 longer freeze-thaw cycles, each of 15 seconds freeze followed by 15 seconds thaw, however, Radmanesh and Azar-Beig (20), used a closed contact $\mathrm{CO}_{2}$ system applied for 10-15 seconds freeze for 8 weeks, with hair regrowth $>50 \%$ in $38.64 \%$ of 44 patients with AA or AT. Recently, Abdel Motaleb and Sayed ${ }^{(37)}$, compared 3 different freezing durations in 2 freeze-thaw cycles of liquid nitrogen, of either $3-5,8-10$, or $13-15$ seconds each, with good to moderate response in $65.2 \%, 76 \%$, and $76.2 \%$, respectively. They concluded that using 8-10 seconds dual freeze-thaw cycles is the optimum and safe timing.

Superficial cryotherapy is contraindicated in patients with intolerance to cold. Adverse effects are usually mild, transient, reversible, and depend on the freezing duration. They include mild pain, pruritus, erythema, edema, blistering, erosion, crustation, and transient pigmentary alteration ${ }^{(19,28)}$. 


\section{Conclusion:}

Superficial cryotherapy can be used as a first-line treatment in mild forms of AA especially in children, as it's less painful and has few and transient side effects than intralesional and topical steroids which are used as first-line therapies. It can be considered a safe alternative method particularly for those who do not respond to topical or intralesional steroids and can be combined with other therapeutic options in various subtypes of AA.

\section{References:}

1-Fricke A and Miteva M: Epidemiology and burden of alopecia areata: a systematic review. Clin Cosmet Investig Dermatol.2015;8:397-403.

2-Mirzoyev S, Schrum A, Davis M, and Torgerson R: Lifetime incidence risk of alopecia areata estimated at $2.1 \%$ by Rochester Epidemiology Project, 19902009. J Invest Dermatol. 2014;134(4):1141-1142.

3-Wolff H, Fischer T, and Blume-Peytavi $\mathrm{U}$ : The Diagnosis and Treatment of Hair and Scalp Diseases. Dtsch Arztebl Int. 2016;113(21):377-386.

4-Kuty-Pachecka M: Psychological and psychopathological factors in alopecia areata. Psychiatr Pol. 2015;49(5):955-964.

5-Strazzulla L, Wang E, Avila L, Lo Sicco K, Brinster N, Christiano A, and Shapiro J: Alopecia areata: An appraisal of new treatment approaches and overview of current therapies. J Am Acad Dermatol. 2018;78(1):15-24.

6-Guttman-Yassky E, Ungar B, Noda S, Suprun M, Shroff A, Dutt R, Khattri S, Min $M$, et al: Extensive alopecia areata is reversed by IL-12/IL-23p40 cytokine antagonism. J Allergy Clin Immunol. 2016;137(1):301-304.

7-Jabbari A, Sansaricq F, Cerise J, Chen J, Bitterman A, UlerioG and Borbon J: An open-label pilot study to evaluate the efficacy of Tofacitinib in moderate to severe patch-type Alopecia Areata, Totalis, and Universalis. $\mathbf{J}$ Invest Dermatol. 2018;138(7):1539-1545.
8-Wang E and Christiano A: The changing landscape of alopecia areata: the translational landscape. Adv Ther. 2017;34(7):1586-1593.

9-Cranwell W, Lai V, Photiou L, Meah N, Wall D, Rathnayake D, Joseph S, Chitreddy $\mathrm{V}$ et al: Treatment of alopecia areata: An Australian expert consensus statement. Australas J Dermatol. 2019;60:163-170.

10-Mesinkovska $\quad \mathrm{N}$ : $\quad$ Emerging Unconventional Therapies for Alopecia Areata. J Investig Dermatol Symp Proc. 2018;19(1):S32-S33.

11-Sharma V and Sujay K: Guidelines for cryotherapy. Indian J Dermatol Venereol Leprol; 2009;75(S2):S90-S100.

12-Lawrence $\mathrm{C}$ and Telfer N: cryotherapy in Burns TB, Cox N, Griffiths C, editors. Rook's Textbook of Dermatology. 8th ed. United Kingdom: Blackwell Publishing. 2010; volume 4. chapter 77. pp.77.39.

13-Yiu W, Basco M, Aruny J, Cheng S and Sumpio B: Cryosurgery A review. Int J Angiol. 2007;16(1):1-6.

14-Winkler J, Jeronimo J, Singleton J, Janmohamed A, and Santos C: Performance of cryotherapy devices using nitrous oxide and carbon dioxide. Int $\mathbf{J}$ Gynaecol Obstet. 2010;111(1):73-77.

15-Costello J, Culligan K, Selfe J, and Donnelly A: Muscle, Skin and Core Temperature after $-110^{\circ} \mathrm{C}$ Cold Air and $8^{\circ} \mathrm{C}$ Water Treatment. PLoS ONE 2012;7(11):e48190.

16. James WD, Berger $\mathrm{T}$, and Elston D: Andrew's diseases of the skin. Clinical dermatology. 9th ed. Elsevier Health Sciences; 2011, volume 10, pp 403-415.

17-Hoffmann NE and Bischof JC: The cryobiology of cryosurgical injury. Urology. 2002;60(2 Suppl 1):40-49.

18-Pimentel C, Moraes A, and Cintra M: Angiogenic effects of cryosurgery with liquid nitrogen on the normal skin of rats, through morphometric study.

A Bras Dermatol. 2014;89(3):410-413.

19-Zawar V and Karad G: Liquid Nitrogen Cryotherapy in Recalcitrant Alopecia Areata: A Study of 11 Patients. Int J Trichology.2016;8(1):15-20.

20-Radmanesh $M$ and Azar Beig M: Cryotherapy as an alternative therapy for 
the treatment of recalcitrant alopecia areata. Iran J Dermatol. 2013;16 (64):49-52.

21-Lindsay A, Othman M, Prebble H, Davies S and Gieseg S: Repetitive cryotherapy attenuates the in vitro and in vivo mononuclear cell activation response. Exp Physiol. 2016;101(7):851-865.

22-Lee B, Lee W, Yoo M and Ahn S: Cryotherapy of alopecia areata. Korean J Dermatol. 1994;32:416-420.

23-Huang P, Huang S and Wei G: Onehundred twenty-three cases of alopecia areata treated with liquid nitrogen cryotherapy. J Clin Dermatol. 1986;15:269. 24- Lei Y, Nie Y, Zhang JM, Liao DY, Li HY, and Man MQ: Effect of superficial hypothermic cryotherapy with liquid nitrogen on alopecia areata. Arch Dermatol 1991;127:1851-1852.

25- Kim TH, Kim DS, and Kim SW: Effect of cryotherapy with liquid

nitrogen on alopecia areata. Korean $\mathbf{J}$ Dermatol 1994;32:421-426.

26-Hong S, Jeon S, Oh T, and Lee W: A retrospective study of the effect of superficial cryotherapy on alopecia areata. Korean J Dermatol. 2006;44(3): 274-280.

27- Faghihi $G$ and Radan M: Jet cryotherapy vs clobetasol propionate lotion in alopecia areata. Skinmed. 2014;12(4):209-211.

28-Jun M, Lee N, and Lee W: Efficacy and safety of superficial cryotherapy alopecia areata: A retrospective, comprehensive review of 353 cases over 22 years. J Dermatol. 2017;44(4):386-933.

29-Amirnia M, Mahmoudi S, KarkonShayan F, Alikhah H, Piri R, NaghaviBehzad M, and Ranjkesh M: Comparative study of intralesional steroid injection and cryotherapy in alopecia areata. Niger Med J. 2015;56(4):249-252.

30-Abdel-Majid E, Abdel-Kader D, and Allam A: Liquid nitrogen cryotherapy in the treatment of alopecia areata: An Egyptian study. J Curr Med Res Pract. 2018;3:187-190.

31-Sayed D, Allam A, and Abdel-Majid E: Superficial cryotherapy versus topical psoralen and ultraviolet $\mathrm{A}$ in the treatment of alopecia areata: a randomized, controlled trial. J Egypt Women's Dermatol Soc. 2020;17:98-103.
32-Lee SJ, Kim JY, Hann SK. Two cases of alopecia areata on eyebrow treated by superficial cryotherapy. Korean J Dermatol 2003;41:1079-1081.

33-Jeon S, Ahn B, Lee S and Lee W: Superficial cryotherapy of alopecia areata in eyebrows. Korean $\mathrm{J}$ Dermatol. 2004;42(8):1024-1027.

34-Jun M and Lee W: Therapeutic Effect of Superficial Cryotherapy on Alopecia Areata: A Prospective, Split-scalp Study in Patients with Multiple Alopecia Patches. Ann Dermatol. 2017;29(6):722-727.

35-Morita K, Nakamura M, Nagamachi M, Kishi T, and Miyachi Y: Seventeen cases of alopecia areata: the combination of SADBE topical immunotherapy with other therapies. J Dermatol 2002;29:661-664.

36- Gita F and Mohammadreza R: Liquid nitrogen cryotherapy vs. betamethasone lotion in the management of alopecia areata. J Clin Med Res.

2013;5:18-22.

37-Abdel Motaleb A and Sayed D: Different freezing time of superficial liquid nitrogen cryotherapy in the treatment of recalcitrant alopecia areata: Randomized clinical trial. Dermatol Ther. 2020;33(4):e13640. 